\title{
Bemerkung zu dem Aufsatze des Herrn B. Igel. "Zur Theorie der Differentialgleichungen."
} (S. 124-130.)

\author{
Von E. Grünfeld in Nikolsburg.
}

Herr B. Igel macht am Schlusse seines Aufsatzes folgende Äaßerung: „Herr Grünfeld kümmert sich um die einfache und einheitliche Form nicht and lässt auch in den Fällen, wo ihm die Ausrechnung gelungen ist, Exponentialfunctionen $z u$, es fehlen daher der Frage alle drei Merkmale und sie hat überhaupt keinen richtigen Sinn."

Diesen Auslassungen gegeniiber bemerke ich Folgendes:

Im 115. Bande des Journals für die reine und angewandte Mathematik habe ich auf zweierlei Art die Formel

$$
D\left(y_{1}, \ldots, y_{n}\right) D\left(u_{1}, \ldots, u_{n}\right)=p_{n}^{n-1}
$$

abgeleitet (S. 332), wo $y_{1}, \ldots, y_{\eta \imath}$ ein Fundamentalsystem von Lösungen der Differentialgleichung

$$
\frac{d^{n} y}{d x^{n}}+p_{1} \frac{d^{n-1} y}{d x^{n-1}}+\cdots+p_{n} y=0
$$

und $u_{1}, \ldots, u_{n}$ ein ebensolches system der zugehörigen Adjungierten der ersten Zeile bedeuten. Die eine Art bestand darin, dass die beiden in $\left(\mathbf{1 3}^{\prime}\right)$ vorkommenden Determinanten direct multipliciert wurden, bei der anderen wurde von dem bekannten Satze Gebrauch gemacht, dass, von einem constunten Factor abgesehen, $D\left(y_{1}, \ldots, y_{n}\right)=e^{-\int p_{1} d x}$ ist. Auf Seite 335, Anfang der Nr. 2, schrieb ich: „Wie ungleich schwieriger es ist, die Beziehung zwisehen den Determinanten $D\left(y_{1}, \ldots, y_{n}\right)$ und $D\left(u_{1}, \ldots, u_{n}\right)$ bei den übrigen Adjungierten durch directe Multiplication ersterer abzuleiten, mag aus einem einfachen Beispiele hervorgehen." In Ausführung dieses Beispieles gelangte ich S. $337 \mathrm{zu}$ der Formel:

$$
D\left(y_{1}, y_{2}, y_{3}\right) \cdot D\left(u_{1}, u_{2}, u_{3}\right)=p_{1} p_{2}-p_{3}+p_{2}^{\prime}
$$

and zeigte alsdann, weil eben die Wertermittelung des Determinantenproductes durch Multiplication zu große Schwierigkeiten darbietet, SS. 337--342, wie dieselbe nach der zweiten erwähnten Methode für alle $n$ Adjungierten bewerkstelligt werden kömne.

Demgemäß ist die Bemerkung des Herm Igel, dass ich um die einfache und einheitliche Form mich nicht gekümmert habe, ganz und gar unbegründet, Ebenso vollständig der Wahrheit ermangelnd ist es auch, wenn er sägt, dass ich auch in den Fällen, wo mir die Ausrechnung gelungen ist, Exponentialfunctionen zulasse, da weder in Formel (13') noch in (24) eine Exponentialgröße vorkommt. Ebenso ergibt sich nach der zweiten Methode, dass für alle $n$ Adjungierten das erwähnte Determinantenproduct sich als rationale Function der Coefficienten $p_{1}, \ldots, p_{n}$ und deren Ableitungen darstellt, welche keine Exponentialfunction einschlieft, wie der aufmerksame Leser sofort erkennt.

Schließlich hebe ich noch hervor, dass Igel's Formel (22) $|\varphi|==\gamma_{3}^{2}$ aus meiner Formel $\left(13^{\prime}\right)$ für $n=3$ hervorgeht, sowio dass seine Formel (23)

$$
|\varphi|=p_{3}-p_{1} p_{2}-p_{2}^{\prime}
$$

vom Factor - 1 abgesehen mit meiner Formel (24) identisch ist und dass er somit die Formeln, die nach seiner Ansicht, überhaupt keinen richtigen Sinn" haben, selbst bringt und durch Vergleichung derselben mit meinen Formeln (13') und (24) sogar zu einer allgemeinen Regel gelangen zu können glaubt. 